\title{
Non-volitional assessment of skeletal muscle strength in patients with chronic obstructive pulmonary disease
}

\author{
W D-C Man, M G G Soliman, D Nikoletou, M L Harris, G F Rafferty, N Mustfa, \\ M I Polkey, J Moxham
}

Thorax 2003;58:665-669

See end of article for authors' affiliations

Correspondence to: Dr W Man, Respiratory Muscle Laboratory, Guy's, King's and St Thomas' School of Medicine, King's College Hospital, Bessemer Road, London SE5 9PJ, UK; William.man@kcl.ac.uk

Revised version received 2 April 2003

Accepted for publication 19 April 2003

\begin{abstract}
Background: Although quadriceps weakness is well recognised in chronic obstructive pulmonary disease (COPD), the aetiology remains unknown. In disabled patients the quadriceps is a particularly underused muscle and may not reflect skeletal muscle function as a whole. Loss of muscle function is likely to be equally distributed if the underlying pathology is a systemic abnormality. Conversely, if deconditioning and disuse are the principal aetiological factors, weakness would be most marked in the lower limb muscles.

Methods: The non-volitional technique of supramaximal magnetic stimulation was used to assess twitch tensions of the adductor pollicis, quadriceps, and diaphragm muscles (TwAP, TwQ, and TwPdi) in 22 stable non-weight losing COPD patients and 18 elderly controls.

Results: Mean (SD) TwQ tension was reduced in the COPD patients (7.1 (2.2) kg v 10.0 (2.7) kg; $95 \%$ confidence intervals $(\mathrm{Cl})-4.4$ to $-1.4 ; \mathrm{p}<0.001$ ). Neither TwAP nor TwPdi (when corrected for lung volume) differed significantly between patients and controls (mean (SD) TwAP 6.52 (1.90) N for COPD patients and $6.80(1.99) \mathrm{N}$ for controls $195 \% \mathrm{Cl}-1.5$ to $0.97, \mathrm{p}=0.65$; TwPdi 23.0 (5.6) $\mathrm{cm} \mathrm{H}_{2} \mathrm{O}$ for COPD patients and 23.5 (5.2) $\mathrm{cm} \mathrm{H}_{2} \mathrm{O}$ for controls (95\% Cl -4.5 to 3.5, $\left.\mathrm{p}=0.81\right)$. Conclusions: The strength of the adductor pollicis muscle (and the diaphragm) is normal in patients with stable COPD whereas quadriceps strength is substantially reduced. Disuse may be the principal factor in the development of skeletal muscle weakness in COPD, but a systemic process preferentially affecting the proximal muscles cannot be excluded.
\end{abstract}

$\mathrm{T}$ here is accumulating evidence of skeletal muscle dysfunction in patients with chronic obstructive pulmonary disease (COPD) which is recognised as contributing to reduced exercise capacity, ${ }^{1}$ impaired quality of life, ${ }^{2}$ and increased health care utilisation. ${ }^{3}$ This has attracted interest because the peripheral muscles represent a potential site for improving patients' level of function despite the largely irreversible impairment of the lungs.

Much research has concentrated on the quadriceps because it is readily accessible and is a primary locomotor muscle. However, it may not be representative of skeletal muscle function as a whole because it is a chronically underused muscle in COPD. Controversy therefore remains over whether the main mechanisms involved in skeletal muscle dysfunction are local or systemic. Analysis of the distribution of muscle weakness in COPD would contribute to an understanding of the underlying aetiological processes. Specifically, loss of muscle function should be equally distributed if the underlying pathology is a generalised process such as inflammation, malnutrition, or derangement of blood gases. Conversely, if chronic inactivity and subsequent disuse atrophy are the predominant aetiological mechanisms, there should be greatest weakness in those muscles with the greatest decrease in activity-that is, the locomotor muscles.

Although previous reports ${ }^{45}$ have sought to address this question, these studies have used measures of maximal voluntary contraction force (MVC) to test the hypothesis. This manoeuvre is not always suitable for patients as the ability to perform a true MVC relies on motivation, cooperation, and functional ability. Although MVC is frequently used in clinical practice, submaximal muscle activation is common ${ }^{6}$ and consequently these studies cannot confidently confirm or refute the hypothesis.

Non-volitional methods of assessing contractility overcome some of the limitations of voluntary manoeuvres but are not commonly performed because of the discomfort and technical difficulty of supramaximal electrical nerve stimulation, especially when repeated measurements or high stimulation frequencies are required. In recent years magnetic stimulation has been introduced in the laboratory and clinical setting, and relatively painless non-volitional methods for assessing the strength of the adductor pollicis, ${ }^{7}$ quadriceps, ${ }^{8}$ and diaphragm ${ }^{9}$ muscles have been described in normal subjects and patients. With these techniques a single supramaximal stimulus is delivered to the motor nerve. The tension or pressure generated has a constant relationship with the maximal tetanic tension, or the true MVC, and therefore accurately reflects strength.

We hypothesise that disuse is an important factor in the aetiology of skeletal muscle dysfunction in COPD, and postulate that quadriceps weakness occurs in the absence of any weakness of the adductor pollicis and diaphragm muscles. To test this hypothesis the strength of the adductor pollicis, quadriceps, and diaphragm muscles were assessed in patients with COPD and a group of age matched controls using the twitch responses elicited by supramaximal magnetic nerve stimulation. Since this technique ensures maximal activation and is independent of patient effort, any differences observed must represent true differences in the contractile properties of the muscles under investigation.

\section{METHODS}

Twenty two outpatients with stable COPD were recruited from a respiratory clinic. Inclusion criteria included a baseline forced expiratory volume in 1 second $\left(\mathrm{FEV}_{1}\right)<50 \%$ predicted, ratio of $\mathrm{FEV}$, to vital capacity (VC) of $60 \%$ or less, $<10 \%$ improvement in $\mathrm{FEV}_{1}$ following a bronchodilator trial, at least 20 pack years of smoking, and no infective exacerbation in the preceding 4 weeks. Drug treatment included inhaled short acting $\beta_{2}$ agonist $(n=22)$, inhaled anticholinergic $(n=14)$, 
Table 1 Anthropometric and lung function measurements in study participants

\begin{tabular}{|c|c|c|c|}
\hline & Healthy elderly $(n=18)$ & COPD patients $(n=22)$ & $p$ value \\
\hline Age (years) & $74.6(6.3)$ & $68.6(8.9)$ & 0.02 \\
\hline Height (m) & $1.66(0.13)$ & $1.67(0.10)$ & 0.91 \\
\hline Weight $(\mathrm{kg})$ & 72.7 (13.3) & $72.8(20.4)$ & 0.99 \\
\hline BMI $\left(\mathrm{kg} / \mathrm{m}^{2}\right)$ & $26.2(3.8)$ & $26.2(6.3)$ & 0.98 \\
\hline Fat free mass $(\mathrm{kg})$ & $44.9(9.0)$ & $47.7(10.4)$ & 0.44 \\
\hline $\mathrm{FEV}_{1}$ (litres) & $2.6(0.4)$ & $0.9(0.3)$ & $<0.0001$ \\
\hline $\mathrm{FEV}_{1}$ (\% pred) & $101(8)$ & $38(13)$ & $<0.0001$ \\
\hline $\mathrm{FEV}_{1} / \mathrm{VC}(\%)$ & 75.6 & 37.6 & $<0.0001$ \\
\hline TLC (\% pred) & - & $116.9(18.0)$ & - \\
\hline RV (\% pred) & - & $186.3(48)$ & - \\
\hline TLCO (\% pred) & - & 43.0 (15.5) & - \\
\hline $\mathrm{PaO}_{2}(\mathrm{kPa})$ & $10.2(1.0)$ & $8.8(1.4)$ & 0.08 \\
\hline $\mathrm{PaCO}_{2}(\mathrm{kPa})$ & $5.5(0.3)$ & $5.4(0.9)$ & 0.81 \\
\hline
\end{tabular}

inhaled long acting $\beta_{2}$ agonist $(n=12)$, inhaled corticosteroid $(n=11)$, maintenance oral corticosteroids $(n=2)$, oral theophylline $(n=1)$, and long term oxygen therapy $(n=1)$. Eighteen healthy elderly volunteers served as controls. All study participants were free from cardiac, rheumatological, or neuromuscular disorders that could limit mobility or reduce muscle strength. The local research ethics committee of King's College Hospital approved the protocol and all participants gave informed consent.

Baseline spirometric parameters, transfer factor using the single breath method (Masterscreen PFT, Jaeger, Hoechberg, Germany), lung volumes obtained from body plethysmography (Auto-link, Morgan Medical, Gillingham, Kent, UK), and arterialised earlobe blood gases were measured in all subjects. Fat free mass (FFM) was determined in 20 patients and 12 controls by bioelectrical impedance analysis using a Bodystat 1500 (Bodystat Ltd, Douglas, UK) and disease and age specific regression equations. ${ }^{11}$ Non-volitional measurements of adductor pollicis and quadriceps strength were made in all subjects. Sixteen COPD patients and 15 healthy elderly controls also consented to the measurement of twitch transdiaphragmatic pressure (TwPdi).

\section{Recording of adductor pollicis strength and electromyogram (EMG)}

Adductor pollicis muscle function was studied using the technique previously described by Harris and colleagues with a modified handboard. ${ }^{7}$ Silver/silver chloride surface electromyogram (EMG) electrodes (Arbo Medical, CT, USA) were placed longitudinally over AP with the earth electrode placed on the tip of the index finger. The supinated hand and forearm were placed in a plastic arm splint that was secured to the handboard with velcro tapes to avoid rotation of the wrist, especially during stimulation. The splint was designed with a window on the medial aspect of the wrist and lower forearm to allow access to the ulnar nerve. The hand was further secured by a padded adjustable metal bar placed on the palm of the hand around which the fingers were comfortably flexed. The thumb was abducted and a metal loop was placed around the proximal phalanx. An inextensible metal chain connected the metal loop to a strain gauge (Strainstall, Cowes, Isle of Wight, UK: range 0-20 kg) which was securely positioned on the handboard so that the chain was perpendicular to both the thumb and strain gauge. The skin was marked to outline the flexor carpi ulnaris tendon along its length and the site of the ulnar styloid. The stimulation point was between the flexor carpi ulnaris tendon and the ulnar artery $2.5 \mathrm{~cm}$ proximal to the intersection of the marked lines. A $43 \mathrm{~mm}$ figure of eight coil powered by a Magstim 200 stimulator (Magstim Co Ltd, Whitland, UK) was positioned against the skin with the focus of the output over the stimulation point. Preliminary studies with changes in orientation of the coil head and alterations in the distance between the metal loop and strain gauge to adjust resting tension were conducted before the commencement of the full study.

To avoid twitch potentiation there was a rest period of 20 minutes before the start of stimulation and a 0.5 minute interval between each twitch. Supramaximality of twitch adductor pollicis (TwAP) responses was confirmed by measuring the force generated and the amplitude of the corresponding compound muscle action potential (CMAP) over a range of magnetic power outputs.

Force and EMG were simultaneously recorded and amplified and the signals were passed via a Powerlab 8s recording unit (ADInstruments, Castle Hill, Australia) to a MacIntosh Powermac 7500 running Chart software (ADInstruments) sampling at $10 \mathrm{kHz}$.

\section{Recording of quadriceps strength and electromyogram (EMG)}

Twitch quadriceps (TwQ) muscle tension was studied using the technique previously described by Polkey and colleagues ${ }^{8}$ with a specially designed chair from which the back was removed and laid flat. The subjects were studied supine with the knee flexed at $90^{\circ}$ over the end of the chair. Surface EMG was recorded using silver/silver chloride electrodes (Arbo Medical) placed over the belly of the rectus femoris. An inextensible strap was placed around the ankle and connected to a strain gauge (Strainstall range $0-100 \mathrm{~kg}$ ) mounted to the back of the chair so that the strap ran perpendicular to the ankle and gauge. A $70 \mathrm{~mm}$ figure of eight coil head (powered by a double Magstim 200 stimulator) was positioned high in the femoral triangle just lateral to the femoral artery; the best spot was determined by minor positional adjustments during stimulations and marked. Potentiation was avoided and supramaximality determined as for the adductor pollicis muscle. Force and EMG were recorded with the same equipment and software set up as for the adductor pollicis.

\section{Measurement of diaphragm strength}

Transdiaphragmatic pressure (Pdi) was recorded by measuring oesophageal pressure (Poes) and gastric pressure (Pga) with a pair of conventionally placed polyethylene balloon catheters. Pressures were measured by differential pressure transducers (Validyne MP45, Validyne, Northridge, CA, USA). The signals from the transducers were connected to an analogue-digital board (NB-MIO-16, National Instruments, Austin, TX, USA) and recorded by a MacIntosh Centis 650 computer using Labview 2.2 software (National Instruments). Twitch transdiaphragmatic pressures (TwPdi) were measured following bilateral anterolateral magnetic stimulation of the 

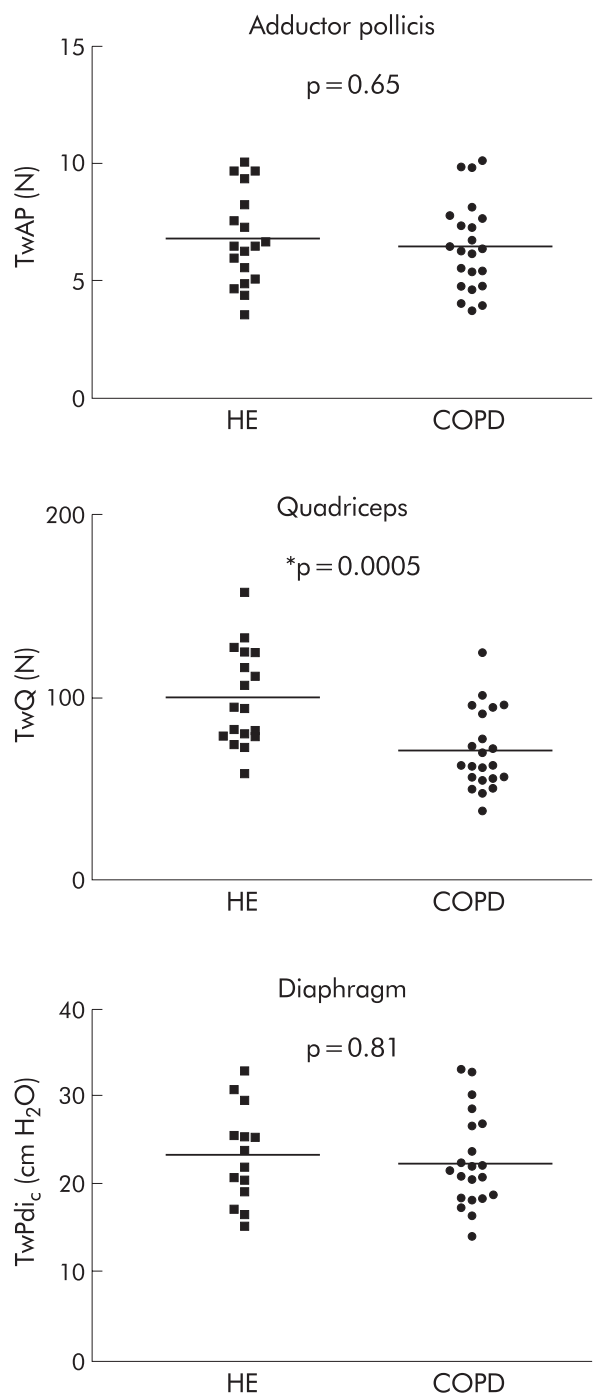

Figure 1 Scatterplots showing individual data and group means of non-volitional skeletal muscle strength in COPD and healthy elderly controls $(\mathrm{HE})$. TwAP=twitch adductor pollicis tension; TwQ $=$ twitch quadriceps tension; $\mathrm{TwPdi}_{c}=$ twitch transdiaphragmatic pressure corrected for lung volume.

phrenic nerves (BAMPS) as previously described. ${ }^{9}$ Supramaximality of the BAMPS technique has been reliably demonstrated in the laboratory setting by our group and others. ${ }^{912} 13$ Even in the challenging ICU setting we have shown that BAMPS was supramaximal in 23 of 25 patients $^{14}$; supramaximality was not specifically re-tested in the current protocol.

\section{Data analysis and statistics}

Predicted values for lung function tests were those of the British Thoracic Society. ${ }^{15}$ The effects of lung volume on TwPdi are well known, so a TwPdi corrected for lung volume (TwPdi $_{c}$ ) at the patients' predicted thoracic gas volume (Vtg) was calculated based on the observation that mean TwPdi decreases at $3.5 \mathrm{~cm} \mathrm{H}_{2} \mathrm{O} / \mathrm{l}$ at volumes above normal Vtg. ${ }^{16}$ For the twitch tensions the mean of five stimulations at $100 \%$ magnetic power output was calculated. Statistical analysis was performed using SPSS 10.1 for Windows (SPSS Ltd, Chicago, IL, USA). Differences between the COPD and control groups were compared using an unpaired $t$ test.

\section{RESULTS}

Anthropometric data for the COPD patients and healthy elderly control subjects are summarised in table 1 . The two groups were well matched for sex, height, weight, BMI, and fat free mass. Supramaximality of TwAP was achieved in 37 of the 40 study participants at a mean magnetic output of $91.6 \%$; for TwQ supramaximality was achieved in 38 out of 40 patients at a mean magnetic output of $90.1 \%$. The individual data for the non-volitional tests of muscle strength are illustrated in fig 1.

Despite a mean $30 \%$ reduction in quadriceps strength in the COPD group (mean (SD) TwQ 7.1 (2.2) kg $v 10.0$ (2.7) kg; 95\% CI -4.4 to $-1.4, \mathrm{p}<0.001$ ), twitch amplitudes of the adductor pollicis muscle were normal (TwAP $6.52(1.90) \mathrm{N}$ and 6.80 (1.99) $\mathrm{N}$ for the COPD patients and elderly controls, respectively; $95 \% \mathrm{CI}-1.5$ to $0.97, \mathrm{p}=0.65$ ). Like the adductor pollicis, diaphragm strength was not reduced in the COPD group (TwPdi 23.0 (5.6) $\mathrm{cm} \mathrm{H}_{2} \mathrm{O}$ for the COPD group and 23.5 (5.2) $\mathrm{cm} \mathrm{H}_{2} \mathrm{O}$ for the controls; $95 \% \mathrm{CI}-4.5$ to $3.5, \mathrm{p}=0.81$ ).

Seven of the COPD patients had taken oral corticosteroids in the preceding 6 months with a mean daily dose of $2.1 \mathrm{mg}$ (two patients were on low dose maintenance corticosteroids and five had received a corticosteroid-burst regimen for infective exacerbations). Subgroup analysis showed no significant difference in TwAP or TwQ between these individuals and the other COPD patients (TwAP $6.95(2.60) \mathrm{N} v 6.32(1.55) \mathrm{N}, 95 \%$ CI -1.20 to 2.47, $\mathrm{p}=0.52$; TwQ $7.06(3.20) \mathrm{kg} v 7.13$ (1.63) kg, $95 \%$ CI -3.0 to $2.9, \mathrm{p}=0.96$ ), and when the patients on corticosteroids were excluded from analysis the significant reduction in quadriceps strength persisted $(95 \%$ CI -4.4 to -1.3 ; $\mathrm{p}<0.001$ ).

\section{DISCUSSION}

This is the first study to compare the strength of the adductor pollicis muscle in COPD patients with age matched controls, and the first to non-volitionally assess the distribution of skeletal muscle weakness. The principal finding is that the strength of the adductor pollicis muscle (and diaphragm) is normal in patients with COPD, despite a substantial reduction in quadriceps strength. This indicates that, for the patients studied, a generalised myopathy does not exist in COPD and the adductor pollicis and diaphragm muscles behave in a different way from the quadriceps.

\section{Methodology}

MVC is often used for routine measurements of muscle strength. A true MVC relies on subject motivation, cooperation, and functional ability-factors particularly relevant in children, patients on intensive care units, those with cognitive difficulties, and those prevented from performing a true MVC by pain. However, even in well motivated subjects, submaximal muscle activation is common in routine clinical practice. ${ }^{6}$ There is therefore a need for non-volitional methods to assess muscle strength in clinical settings.

The true strength of a muscle is the maximum tetanic tension, which requires the delivery of trains of stimuli at different frequencies and construction of a force-frequency curve. For many muscles this is not tolerable in humans, hence techniques using single supramaximal stimuli have been developed. The tension generated enjoys a constant relationship with the maximal tetanic tension. Magnetic stimulation has recently been successfully introduced to the clinical and laboratory setting to assess the strength of the adductor pollicis ${ }^{7}$ and quadriceps muscles ${ }^{8}$ and the diaphragm. ${ }^{9}$ This form of stimulation is more reliable in ensuring supramaximality and is less painful than traditional electrical stimulation, so is more acceptable to patients; in this study magnetic stimulation was well tolerated by all participants.

\section{Significance of findings}

The strength of the adductor pollicis muscle in patients with COPD has not previously been compared with that of elderly control subjects, although Whittaker et $a l^{17}$ reported that short term refeeding of malnourished COPD patients did not 
improve adductor pollicis muscle function. By comparing their data with historical controls, Whittaker and colleagues concluded that adductor pollicis function was normal in COPD. The TwPdi data in the present study support previous observations that, when corrected for lung volume, the contractility of the diaphragm is not reduced in COPD. ${ }^{16}{ }^{18}$ The preservation of strength in the diaphragm may be due to an involuntary "training" effect derived from the high work of breathing in patients with COPD. Consistent with this observation, the diaphragm muscle in COPD shows an increase in oxidative capacity, ${ }^{19}$ and there is a fast-to-slow fibre type transformation-that is, an increase in type I fibres ${ }^{20}$ consistent with "overtraining".

Although skeletal muscle dysfunction is well recognised in COPD, the relative contributions of systemic factors (such as inflammation, hypoxia, acidosis, malnutrition) and local muscle factors (such as deconditioning) remain unknown. One plausible explanation for the findings in this study is disuse atrophy. As the disease progresses and symptoms intensify, patients become locked into a downward spiral of breathlessness, leading to inactivity, and subsequent muscle deconditioning with the greatest effect on the muscles that are least used-that is, the quadriceps muscle. In comparison, the adductor pollicis muscle is continually used for activities of daily living such as eating, grooming, and writing. This hypothesis is supported by previous studies (using volitional techniques) showing the unequal distribution of muscle weakness in COPD, with the strength of the upper extremity muscles being relatively preserved compared with that of the lower extremity muscles. ${ }^{45}$

Apart from distribution of muscle weakness, deconditioning shares many similarities with COPD in its effects on the morphological and metabolic characteristics of skeletal muscle. Alterations in the distribution of muscle fibre type as a result of chronic inactivity ${ }^{21}$ are similar to those observed in patients with COPD, with a reduction in slow twitch type I fibres and an increase in fast twitch type II fibres. ${ }^{22}$ In addition, a reduction in oxidative metabolism is found in the quadriceps muscle of subjects with deconditioning ${ }^{21}{ }^{23}$ as well as in patients with COPD. ${ }^{24}$ Consistent with our hypothesis, skeletal muscles not primarily used for locomotion (such as tibialis anterior and deltoid) do not show reduced oxidative capacity in COPD. ${ }^{25}{ }^{26}$ In a recent comprehensive review of the literature Franssen and colleagues compared the alterations in peripheral skeletal muscle function, muscle morphology, and energy metabolism in COPD with those of reduced physical activity. ${ }^{27}$ The authors concluded that "deconditioning of peripheral muscles is very likely to be an important contributor to the alterations in peripheral skeletal muscles that occur in patients with COPD". Despite the similarities in skeletal muscle abnormalities between reduced activity and chronic lung disease, atrophy in disuse principally affects type I fibres ${ }^{28}$ whereas type IIx fibres seem to be preferentially affected in $\mathrm{COPD}^{29}$; however, there are published data demonstrating type II atrophy in disuse ${ }^{2123}$ and type I atrophy in COPD. ${ }^{22}$

Our hypothesis is dependent on the assumption that the physical activity level of our cohort of COPD patients was reduced compared with our healthy elderly group. We believe this to be a reasonable assumption as published data confirm that activity levels are decreased in patients with COPD compared with age matched healthy controls. ${ }^{30}{ }^{31}$ Furthermore, if our hypothesis that detraining is a significant contributor to skeletal muscle weakness is correct, exercise training would be expected to improve muscle function. It is now well accepted that pulmonary rehabilitation improves exercise capacity and quality of life in COPD without changes in lung function. ${ }^{32}$ Exercise training has been shown to increase volitional and non-volitional quadriceps strength, ${ }^{33}$ to increase skeletal muscle oxidative capacity, and reduce lactic acidosis. ${ }^{35}$ Another treatment that "trains" muscles-neuromuscular electrical stimulation-has been shown to improve quadriceps strength, exercise capacity, and quality of life. ${ }^{36}$
An alternative explanation for the results of this study is that a systemic process does operate but preferentially affects the proximal muscles; steroid myopathy, although excluded as a cause in the present study, is an example of such a process. This is supported by work by Gosselink and colleagues ${ }^{4}$ who reported that handgrip and elbow flexion force are less affected than shoulder abduction or knee extension force in patients with COPD using volitional techniques. Why a systemic inflammatory process (or, indeed, steroid myopathy) should have a predilection for proximal muscles is not known. A possible explanation is that some myopathies preferentially affect certain fibre types; necroscopic data suggest that the adductor pollicis muscle, although containing both fibre types, is largely composed of type 1 fibres ${ }^{37}$ whereas the quadriceps muscle consists of $43 \%$ type 1 fibres and 57\% type 2 fibres. ${ }^{38}$ Non-volitional assessment of a large proximal upper limb muscle and a small distal lower limb muscle would help to confirm or refute this hypothesis, but well established reproducible techniques are not currently available.

In summary, the strength of the adductor pollicis muscle (and the diaphragm) is normal in patients with stable COPD despite the presence of significant weakness of the quadriceps. This observation supports the hypothesis that deconditioning and disuse atrophy are important in the development of muscle weakness in patients with COPD, at least in those who are stable without recent weight loss. However, a systemic process preferentially affecting the proximal muscles cannot be excluded. Further studies are required to delineate whether disuse alone is a sufficient condition, or whether interaction with systemic factors is also required.

\section{Authors' affiliations}

W D-C Man, M G G Soliman, D Nikoletou, M L Harris, G F Rafferty, N Mustfa, J Moxham, Respiratory Muscle Laboratory, Guy's, King's and St Thomas' School of Medicine, King's College Hospital, London, UK M I Polkey, Royal Brompton Hospital, London, UK

William Man is a Clinical Research Training Fellow of the Medical Research Council (UK).

\section{REFERENCES}

1 Gosselink R, Troosters T, Decramer M. Peripheral muscle weakness contributes to exercise limitation in COPD. Am J Respir Crit Care Med 1996;153:976-80.

2 Simpson K, Killian K, McCartney N, et al. Randomised controlled trial of weightlifting exercise in patients with chronic airflow limitation. Thorax 1992;47:70-5

3 Decramer M, Gosselink R, Troosters T, et al. Muscle weakness is related to utilization of health care resources in COPD patients. Eur Respir J 1997; 10:417-23

4 Gosselink R, Troosters T, Decramer M. Distribution of muscle weakness in patients with stable chronic obstructive pulmonary disease. $J$ Cardiopulm Rehabil 2000:20:353-60.

5 Bernard S, LeBlanc P, Whittom F, et al. Peripheral muscle weakness in patients with chronic obstructive pulmonary disease. Am J Respir Crit Care Med 1998;158:629-34.

6 Allen GM, Gandevia SC, McKenzie DK. Reliability of measurements of muscle strength and voluntary activation using twitch interpolation. Muscle Nerve 1995; 18:593-600.

7 Harris ML, Luo YM, Watson AC, et al. Adductor pollicis twitch tension assessed by magnetic stimulation of the ulnar nerve. Am J Respir Crit Care Med 2000;162:240-5.

8 Polkey MI, Kyroussis D, Hamnegard CH, et al. Quadriceps strength and fatigue assessed by magnetic stimulation of the femoral nerve in man. Muscle Nerve 1996;19:549-55.

9 Mills GH, Kyroussis D, Hamnegard CH, et al. Bilateral magnetic stimulation of the phrenic nerves from an anterolateral approach. Am J Respir Crit Care Med 1996;154:1099-105.

10 Deurenberg $\mathbf{P}$, van der Kooij K, Evers $\mathrm{P}$, et al. Assessment of body composition by bioelectrical impedance in a population aged greater than 60 y. Am J Clin Nutr 1990;51:3-6.

11 Steiner MC, Barton RL, Singh SJ, et al. Bedside methods versus dual energy $\mathrm{X}$-ray absorptiometry for body composition measurement in COPD. Eur Respir J 2002;19:626-31.

12 Man WD, Luo YM, Mustfa N, et al. Postprandial effects on twitch transdiaphragmatic pressure. Eur Respir J 2002;20:577-80.

13 Mador MJ, Khan S, Kufel TJ. Bilateral anterolateral magnetic stimulation of the phrenic nerves can detect diaphragmatic fatigue. Chest $2002 ; 121: 452-8$. 
14 Watson AC, Hughes PD, Harris ML, et al. Measurement of twitch transdiaphragmatic, esophageal, and endotracheal tube pressure with bilateral anterolateral magnetic phrenic nerve stimulation in patients in the intensive care unit. Crit Care Med 2001;29:1325-31.

15 Anon. Guidelines for the measurement of respiratory function. Recommendations of the British Thoracic Society and the Association of Respiratory Technicians and Physiologists. Respir Med 1994;88:165-94.

16 Polkey MI, Kyroussis D, Hamnegard CH, et al. Diaphragm strength in chronic obstructive pulmonary disease. Am J Respir Crit Care Med 1996:154:1310-7.

17 Whittaker JS, Ryan CF, Buckley PA, et al. The effects of refeeding on peripheral and respiratory muscle function in malnourished chronic obstructive pulmonary disease patients. Am Rev Respir Dis 1990;142:283-8.

18 Similowski T, Yan S, Gauthier AP, et al. Contractile properties of the human diaphragm during chronic hyperinflation. N Engl J Med 1991;325:917-23

19 Levine S, Gregory C, Nguyen T, et al. Bioenergetic adaptation of individual human diaphragmatic myofibers to severe COPD. J App Physiol 2002;92:1205-13.

20 Levine S, Kaiser L, Leferovich J, et al. Cellular adaptations in the diaphragm in chronic obstructive pulmonary disease. N Engl J Med 1997;337: 1799-806.

21 Hikida RS, Gollnick PD, Dudley GA, et al. Structural and metabolic characteristics of human skeletal muscle following 30 days of simulated microgravity. Aviat Space Environ Med 1989;60:664-70.

22 Whittom F, Jobin J, Simard PM, et al. Histochemical and morphological characteristics of the vastus lateralis muscle in patients with chronic obstructive pulmonary disease. Med Sci Sports Exerc 1998;30:146774.

23 Klausen K, Andersen LB, Pelle I. Adaptive changes in work capacity, skeletal muscle capillarization and enzyme levels during training and detraining. Acta Physiol Scand 1981;113:9-16.

24 Maltais F, Simard AA, Simard C, et al. Oxidative capacity of the skeletal muscle and lactic acid kinetics during exercise in normal subjects and in patients with COPD. Am J Respir Crit Care Med 1996; 153:288-93

25 Pouw EM, Koerts-de Lang E, Gosker HR, et al. Muscle metabolic status in patients with severe COPD with and without long-term prednisolone. Eur Respir J 2000;16:247-52.
26 Gea JG, Pasto M, Carmona MA, et al. Metabolic characteristics of the deltoid muscle in patients with chronic obstructive pulmonary disease. Eur Respir J 2001;17:939-45.

27 Franssen FM, Wouters EF, Schols AM. The contribution of starvation, deconditioning and ageing to the observed alterations in peripheral skeletal muscle in chronic organ diseases. Clin Nutr 2002;21:1-14

28 Berg HE, Larsson L, Tesch PA. Lower limb skeletal muscle function after 6 wk of bed rest. J Appl Physiol 1997;82:182-8.

29 Gosker HR, Engelen MP, van Mameren H, et al. Muscle fiber type IIX atrophy is involved in the loss of fat-free mass in chronic obstructive pulmonary disease. Am J Clin Nutr 2002;76:113-9.

30 Schonhofer B, Ardes P, Geibel M, et al. Evaluation of a movement detector to measure daily activity in patients with chronic lung disease. Eur Respir J 1997;10:2814-9.

31 Singh S, Morgan MD. Activity monitors can detect brisk walking in patients with chronic obstructive pulmonary disease. J Cardiopulm Rehabil 2001;21:143-8.

32 Griffiths TL, Burr ML, Campbell IA, et al. Results at 1 year of outpatient multidisciplinary pulmonary rehabilitation: a randomised controlled trial. Lancet 2000;355:362-8.

33 O'Donnell DE, McGuire M, Samis L, et al. General exercise training improves ventilatory and peripheral muscle strength and endurance in chronic airflow limitation. Am J Respir Crit Care Med 1998;157:148997.

34 Mador MJ, Kufel TJ, Pineda LA, et al. Effect of pulmonary rehabilitation on quadriceps fatiguability during exercise. Am J Respir Crit Care Med $2001 ; 163: 930-5$

35 Maltais $\mathbf{F}$, LeBlanc $P$, Simard $C$, et al. Skeletal muscle adaptation to endurance training in patients with chronic obstructive pulmonary disease. Am J Respir Crit Care Med 1996;154:442-7.

36 Neder JA, Sword D, Ward SA, et al. Home based neuromuscular electrical stimulation as a new rehabilitative strategy for severely disabled patients with chronic obstructive pulmonary disease (COPD). Thorax 2002;57:333-7.

37 Round JM, Jones DA, Chapman SJ, et al. The anatomy and fibre type composition of the human adductor pollicis in relation to its contractile properties. J Neurol Sci 1984;66:263-72.

38 Johnson MA, Polgar J, Weightman D, et al. Data on the distribution of ibre types in thirty-six human muscles. An autopsy study. J Neurol Sci 1973;18:111-29.

\section{LUNG ALERT}

\section{Prophylactic itraconazole may decrease the rate of invasive fungal infection in allogeneic haematopoietic stem cell transplant recipients}

- Winston DJ, Maziarz RT, Chandrasekar PH, et al. Intravenous and oral itraconazole versus intravenous and oral fluconazole for long-term antifungal prophylaxis in allogeneic hematopoietic stem-cell transplant recipients: a multicenter, randomized trial. Ann Intern Med 2003;138:705-13

nvasive fungal infections account for the majority of deaths due to infection in patients following allogeneic hematopoietic stem cell transplants, although toxicity secondary to antifungal agents and the emergence of resistant fungal strains are presenting clinical dilemmas.

This multicenter randomised open label trial compared prophylactic itraconazole (intravenous and oral) with fluconazole (intravenous and oral) in 140 patients over the age of 13 on the first day after transplantation and continued for 100 days with follow up until day 180 after transplantation or death. The primary end point was the incidence of invasive fungal infection and the secondary end points were the rates of superficial fungal infection, adverse events secondary to the study drug, mortality from fungal infection, and survival.

The incidence of invasive fungal infection was significantly lower in the patients given prophylactic itraconazole with lower though non-significant differences in mortality. Overall, the incidence of invasive fungal infection was high in this study compared with that of earlier studies. This was attributed both to the high use of corticosteroids $(85 \%)$ which is associated with an increased risk of fungal infection and the generally increasing incidence reported throughout transplant centres in the United States.

The safe and effective use of itraconazole in patients undergoing allogeneic haematopoietic stem cell transplantation may represent an important advance in the prevention of possibly fatal invasive fungal infection, although gastrointestinal side effects were significantly higher in patients treated with itraconazole. 